\title{
Optimization of Dalbavancin in Patients with Hepatic or Renal Impairment
}

\author{
Ying Wang1, Jingyi Zhao², Yinhui Yao1* ${ }^{*}$ Junhui Hu${ }^{1}$, Jiuli Hu${ }^{1}, \mathrm{Xu} \mathrm{Xiao}^{1}$, Yanwu Zhao ${ }^{*}$ \\ ${ }^{1}$ Department of Pharmacy, Affiliated Hospital of Chengde Medical College, Chengde, China \\ ${ }^{2}$ Department of Functional Center, Chengde Medical College, Chengde, China \\ Email: *yhyaodrdr@163.com, ${ }^{*}$ cyfyzyw@163.com
}

How to cite this paper: Wang, Y., Zhao, J.Y., Yao, Y.H., Hu, J.H., Hu, J.L., Xiao, X. and Zhao, Y.W. (2020) Optimization of Dalbavancin in Patients with Hepatic or Renal Impairment. Pharmacology \& Pharmacy, 11, 29-37.

https://doi.org/10.4236/pp.2020.112004

Received: December 16, 2019

Accepted: February 25, 2020

Published: February 28, 2020

Copyright $\odot 2020$ by author(s) and Scientific Research Publishing Inc. This work is licensed under the Creative Commons Attribution International License (CC BY 4.0).

http://creativecommons.org/licenses/by/4.0/

\begin{abstract}
Dalbavancin is a novel semi-synthetic glycopeptide antibiotic. In this study, we aimed to optimize the dosage regimen of dalbavancin in patients with hepatic or renal impairment by Mote Carlo simulation. Pharmacokinetic parameters and microbiological data were collected about dalbavancin. 10,000 patients with renal or hepatic impairment analyzed by Crystal Ball to calculate probability of target attainment (PTA) and cumulative fraction of response (CFR). We found that all bacterial PTA and CFR were more than $90 \%$ for dalbavancin in patients with hepatic or renal impairment, except for Enterococcus faecium. There is no need to adjust the dosage regimen of dalbavancin in patients with hepatic or renal impairment.
\end{abstract}

\section{Keywords}

Mote Carlo Simulation, Dalbavancin, Hepatic Impairment, Renal Impairment

\section{Introduction}

Dalbavancin is a novel semi-synthetic glycopeptide antibiotic currently used clinically to treat infections of skin and soft tissues caused by Gram-positive bacteria [1]. Unlike other glycopeptide antibiotics such as vancomycin and teicoplanin, which are excreted almost exclusively through kidney [2] [3], the excretion of dalbavancin includes renal and non-renal pathways of elimination. Therefore, it is necessary to optimize the dosage regimen of dalbavancin in patients with hepatic or renal impairment, so as to further improve the efficacy of the drug and reduce adverse reactions.

In order to maximize the clinical effect of antibiotics and reduce the possibility of antibiotic resistance, we used the Monte Carlo simulation (MCS) method. 
MCS is a very useful tool for dose decision-making in most therapeutic areas [4] [5] [6], not only in the application of multiple antimicrobials, but also in MIC breakpoints [7] [8] [9]. Therefore, in this study, MCS was performed to study the dosage effect of dalbavancin in patients with different hepatic or renal impairment, so as to determine the optimal dosage regimen.

\section{Materials and Methods}

\subsection{Pharmacokinetic Parameters}

PK data for dalbavancin in patients with different hepatic and renal functions are from the literature [10], with the specific data shown in Table 1 and Table 2. Hepatic function damage was classified into mild (Grade A), moderate (Grade B), and severe (Grade C) according to the Child-Pugh classification for Severity of liver disease. Renal impairment was divided into mild (creatinine clearance; $\left.\mathrm{CL}_{\mathrm{CR}}: 50-80 \mathrm{ml} / \mathrm{min}\right)$, moderate $\left(\mathrm{CL}_{\mathrm{CR}}: 30-49 \mathrm{ml} / \mathrm{min}\right)$, severe $\left(\mathrm{CL}_{\mathrm{CR}}:<30\right.$ $\mathrm{ml} / \mathrm{min}$ ) and patients with advanced kidney disease dialysis. The protein binding rate of dalbavancin is $93 \%$, so the free percentage (f) is $7 \%$.

\subsection{Microbial Information}

The minimum inhibitory concentration (MIC)data of dalbavancin for 11 species of bacteria was from the report of the Sentinel Surveillance Program for Antimicrobial Resistance in the U.S. 2011 (shown in Table 3), and the broth microdilution method was determined by the Clinical and Laboratory Standards Institute [11].

\subsection{Monte Carlo Simulation}

The ratio of the area under the concentration time curve (AUC) and the MIC, AUC24 h/MIC, is an important parameter for evaluating the efficacy of dalbavancin [12]. The calculation formula is as follows:

$$
f A U C_{24 \mathrm{~h}} / M I C=(f \times \text { dose }) /(C L \times M I C)
$$

According to the above formula, the acquisition probability of the target value of Pharmacokinetic/pharmacodynamic (PK/PD) is calculated, that is, the probability of target attainment (PTA). The expected probability of the corresponding strain to the target value was calculated according to the following formula, and marked as cumulative fraction of response (CFR):

Table 1. Pharmacokinetic parameters of Dalbavancin in patients with different grades of hepatic impairment.

\begin{tabular}{ccccc}
\hline \multirow{2}{*}{ Parameter } & Normal $(\mathrm{n}=9)$ & \multicolumn{3}{c}{ Hepatic Impairment } \\
\cline { 3 - 5 } & & Mild $(\mathrm{n}=6)$ & Moderate $(\mathrm{n}=6)$ & Severe $(\mathrm{n}=5)$ \\
\hline Dose $(\mathrm{mg} / \mathrm{d})$ & 1000 & 1000 & 1000 & 1000 \\
CL $(\mathrm{L} / \mathrm{h})$ & $0.047 \pm 0.011$ & $0.047 \pm 0.008$ & $0.065 \pm 0.010$ & $0.074 \pm 0.020$ \\
\hline
\end{tabular}


Table 2. Pharmacokinetic parameters of Dalbavancin in patients with different grades of renal impairment.

\begin{tabular}{cccccccc}
\hline \multirow{2}{*}{ Parameter } & $\begin{array}{c}\text { Normal } \\
(\mathrm{n}=9)\end{array}$ & $\begin{array}{c}\text { Mild } \\
(\mathrm{n}=6)\end{array}$ & $\begin{array}{c}\text { Moderate } \\
(\mathrm{n}=6)\end{array}$ & $\begin{array}{c}\text { Severe } \\
(\mathrm{n}=6)\end{array}$ & $\begin{array}{c}\text { Severe } \\
(\mathrm{n}=4)\end{array}$ & $\begin{array}{c}\text { Predialysis } \\
(\mathrm{n}=3)\end{array}$ & $\begin{array}{c}\text { Postdialysis } \\
(\mathrm{n}=3)\end{array}$ \\
\hline Dose & 1000 & 1000 & 1000 & 500 & 1000 & 500 & 500 \\
$(\mathrm{mg} / \mathrm{d})$ & & & & & & \\
$\mathrm{CL}(\mathrm{L} / \mathrm{h})$ & $0.042 \pm$ & $0.038 \pm$ & $0.027 \pm$ & $0.022 \pm$ & $0.024 \pm$ & $0.026 \pm$ \\
& 0.008 & 0.005 & 0.005 & 0.006 & 0.007 & 0.006 & $0.035 \pm 0.011$ \\
\hline
\end{tabular}

Table 3. MIC values of Dalbavancin against 11 strains of bacteria.

\begin{tabular}{ccccccccccccc}
\hline \multicolumn{10}{c}{$\mathrm{MIC}(\mu \mathrm{g} / \mathrm{ml})$} \\
\hline 0.02 & 0.004 & 0.008 & 0.016 & 0.032 & 0.064 & 0.125 & 0.25 & 0.5 & 1 & 2 & 4 & 8 \\
\hline 0 & 0 & 0 & 113 & 1231 & 632 & 58 & 3 & 2 & 3 & 7 & 12 & 10 \\
0 & 0 & 0 & 22 & 88 & 178 & 102 & 30 & 18 & 21 & 16 & 41 & 120 \\
0 & 0 & 10 & 366 & 10,805 & 16,195 & 1502 & 309 & 41 & 17 & 2 & 0 & 0 \\
0 & 0 & 20 & 925 & 1944 & 2173 & 837 & 339 & 84 & 25 & 12 & 1 & 0 \\
0 & 0 & 0 & 483 & 1225 & 316 & 76 & 31 & 4 & 8 & 0 & 0 & 0 \\
0 & 0 & 0 & 17 & 30 & 95 & 171 & 39 & 9 & 7 & 3 & 0 & 0 \\
0 & 0 & 0 & 56 & 106 & 34 & 12 & 0 & 0 & 0 & 0 & 0 & 0 \\
1 & 1 & 50 & 290 & 267 & 162 & 59 & 18 & 0 & 0 & 0 & 0 & 0 \\
6 & 23 & 435 & 1174 & 89 & 53 & 9 & 1 & 0 & 0 & 0 & 0 & 0 \\
0 & 0 & 68 & 105 & 13 & 8 & 1 & 0 & 0 & 0 & 0 & 0 & 0 \\
0 & 0 & 0 & 162 & 72 & 77 & 19 & 2 & 1 & 0 & 0 & 0 & 0 \\
\hline
\end{tabular}

$P T A i$ is the probability of estimating the target value at a specific MIC; $F i$ is the probability of each MIC distribution under a certain bacterial sample size. Therefore, our study determined a reasonable dose of dalbavancin in patients with renal or hepatic impairment when PTA achieves the highest (>90\%) or CFR achieves the best $(>90 \%)$.

Monte Carlo simulations were performed by using Crystal Ball software (version 11.1.2.4.600, Oracle). The PK parameter clearance (CL) is lognormal distributed. The dose was how much given to patients in $24 \mathrm{~h}(\mathrm{mg})$ and $f$ is uniformly distributed. The MIC is subject to a custom distribution. The previous studies showed the best antibacterial effect with fAUC24 h/MIC $\geq 300$ as the target value, so the present study generated 10,000 simulated patients with hepatic and renal impairment to obtain PTA and CFR [13] [14].

\section{Results}

\subsection{PTA}

From the Monte Carlo simulation of dalbavancin for patients with renal and hepatic impairment, PTA values with fAUC24 h/MIC $\geq 300$ as target value at 
different MIC values were shown in Figure 1 and Figure 2. As shown in the Figure 1, the concentration and clearance of dalbavancin decrease and increase with changes in the level of hepatic impairment (Grade B-C), respectively. For patients with hepatic impairment, PTA $>90 \%$ results showed that the highest MIC value for different levels of hepatic impairment was $2 \mu \mathrm{g} / \mathrm{mL}$. As shown in Figure 2, the dalbavancin concentration was significantly increased in patients with severe renal impairment. In patients with advanced renal impairment, although the clearance rate before and after drug dialysis had increased slightly, PTA $>90 \%$ results showed that the highest MIC value was $0.5 \mu \mathrm{g} / \mathrm{mL}$.

\subsection{CFR}

From the Monte Carlo simulation of dalbavancin for patients with renal and hepatic impairment, CFR values with fAUC24 h/MIC $\geq 300$ as the target value at different MIC values were shown in Table 4 and Table 5. Results showed that except for the poor effect on Enterococcus faecium, the dosage of dalbavancin in patients with renal and hepatic impairment reached the target value for other bacteria (Table 4 and Table 5).

\section{Discussion}

As an FDA-approved drug to treat acute bacterial infections of skin and soft tissues, dalbavancin has an initial dose of $1000 \mathrm{mg} / \mathrm{d}$ and a sustained dose of 500 $\mathrm{mg} / \mathrm{d}$ on day 8 [15]. Clinical studies have reported that dalbavancin has linear and dose-related pharmacokinetics with a half-life of $346 \mathrm{~h}$ [10] [14] [16]. About $33 \%$ of dalbavancin was excluded from the urine as a prototype, indicating that non-renal metabolic pathways are important in dalbavancin [10]. In this study, Monte Carlo simulation was used to analyze whether the dose of dalbavancin in patients with hepatorenal impairment reached the target value of $\mathrm{PK} / \mathrm{PD}$ so that

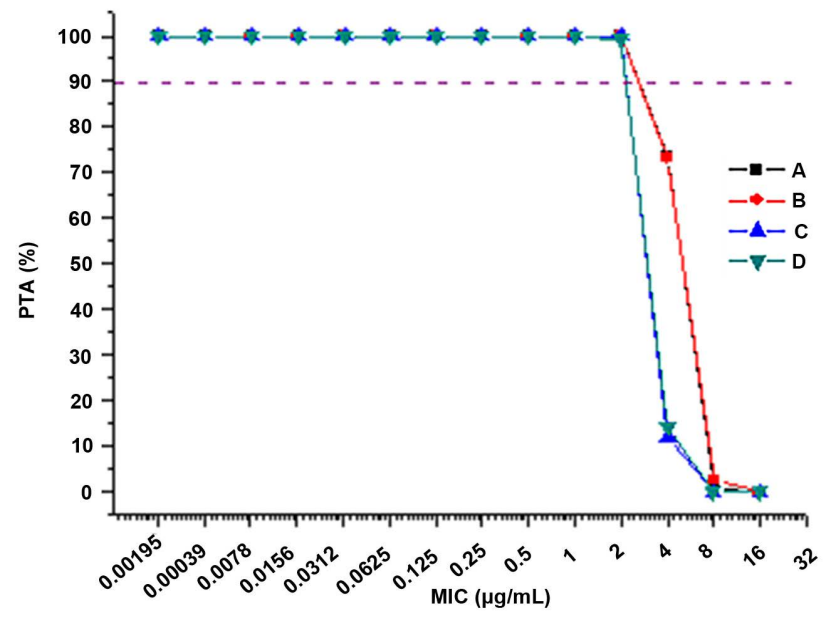

Figure 1. PTA of dalbavancin estimated at different MIC values in patients with hepatic impairment. Different letters represent different grades of liver injury. A, normal; B, mild; C, moderate and $\mathrm{D}$, severe. 


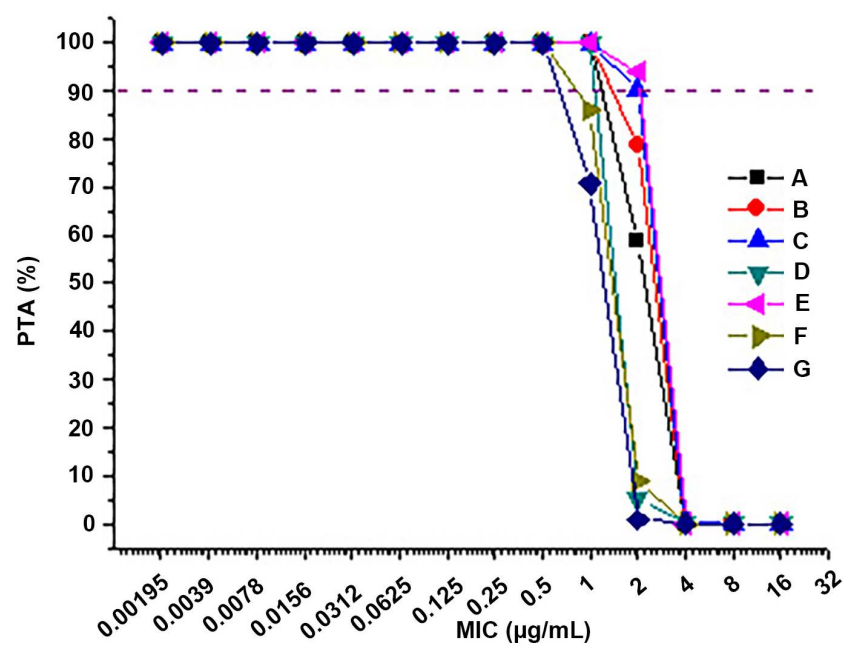

Figure 2. PTA of dalbavancin estimated at different MIC values in patients with renal impairment. Different letters represent different grades of renal injury and the corresponding dosages. A, normal (1000 mg/d); B, mild (1000 mg/d); C, moderate (1000 $\mathrm{mg} / \mathrm{d}) ; \mathrm{D}$, severe $(500 \mathrm{mg} / \mathrm{d}) ; \mathrm{E}$, severe $(1000 \mathrm{mg} / \mathrm{d}) ; \mathrm{F}$, predialysis $(500 \mathrm{mg} / \mathrm{d})$, and $\mathrm{G}$, postdialysis $(500 \mathrm{mg} / \mathrm{d})$.

Table 4. CFR (\%) of dalbavancin in patients with hepatic impairment.

\begin{tabular}{ccccc}
\hline \multirow{2}{*}{ Strains } & \multicolumn{4}{c}{ Hepatic impairment (Dosage (mg/d)) } \\
\cline { 2 - 5 } & Normal (1000) & Mild (1000) & Moderate (1000) & Severe (1000) \\
\hline E. faecalis & 92.91 & 92.91 & 92.57 & 92.58 \\
E. faecium & 61.85 & 62.06 & 58.59 & 58.70 \\
Staphylococcus pyogenes aureus & 99.99 & 99.99 & 99.99 & 99.99 \\
Coagulase-negative staphylococci & 99.99 & 99.99 & 99.99 & 99.99 \\
Staphylococcus epidermidis & 100 & 100 & 100 & 100 \\
Staphylococcus haemolyticus & 99.99 & 99.99 & 99.99 & 99.99 \\
Staphylococcus hominis & 100 & 100 & 100 & 100 \\
Streptococcus agalactiae & 100 & 100 & 100 & 100 \\
Streptococcus pyogenes & 100 & 100 & 100 & 100 \\
Group G Streptococcus & 100 & 100 & 100 & 100 \\
Streptococcus viride & 100 & 100 & 100 & 100 \\
\hline
\end{tabular}

patients can achieve better therapeutic effect. The simulation results show that the given dose of dalbavancin reaches the target value of PK/PD for allpatients with hepatorenal impairment, except for those who has Enterococcus faecium infection. This is consistent with the previous report that the antibacterial spectrum of dalbavancin includes methicillin-sensitive Staphylococcus aureus, methicillin-resistant Staphylococcus aureus, coagulase-negative Staphylococci and Streptococci [17]. Salem A H et al. [18] reported that patients with moderate renal impairment treated with dalbavancin (initial dose $1000 \mathrm{mg} / \mathrm{d}$, subsequent 
Table 5. CFR (\%) of dalbavancin in patients with renal impairment.

\begin{tabular}{|c|c|c|c|c|c|c|c|}
\hline \multirow[b]{2}{*}{ Strains } & \multicolumn{7}{|c|}{ Renal Injury (Dosage (mg/d)) } \\
\hline & $\begin{array}{c}\text { Normal } \\
(1000)\end{array}$ & $\begin{array}{l}\text { Mild } \\
(1000)\end{array}$ & $\begin{array}{c}\text { Moderate } \\
(1000)\end{array}$ & $\begin{array}{c}\text { Severe } \\
(500)\end{array}$ & $\begin{array}{l}\text { Severe } \\
(1000)\end{array}$ & $\begin{array}{c}\text { Predialysis } \\
(500)\end{array}$ & $\begin{array}{c}\text { Postdialysis } \\
\quad(500)\end{array}$ \\
\hline E. faecalis & 92.37 & 92.44 & 92.48 & 92.19 & 92.49 & 92.19 & 92.15 \\
\hline E. faecium & 57.19 & 57.58 & 57.83 & 55.95 & 57.88 & 55.86 & 55.30 \\
\hline Staphylococcus pyogenes aureus & 99.99 & 99.99 & 99.99 & 99.98 & 99.99 & 99.97 & 99.69 \\
\hline Coagulase-negative staphylococci & 99.91 & 99.94 & 99.97 & 99.77 & 99.97 & 99.75 & 99.68 \\
\hline Staphylococcus epidermidis & 100 & 100 & 100 & 99.97 & 100 & 99.94 & 99.89 \\
\hline Staphylococcus haemolyticus & 99.66 & 99.83 & 99.92 & 99.09 & 99.95 & 98.99 & 98.64 \\
\hline Staphylococcus hominis & 100 & 100 & 100 & 100 & 100 & 100 & 100 \\
\hline Streptococcus agalactiae & 100 & 100 & 100 & 100 & 100 & 100 & 100 \\
\hline Streptococcus pyogenes & 100 & 100 & 100 & 100 & 100 & 100 & 100 \\
\hline Group G Streptococcus & 100 & 100 & 100 & 100 & 100 & 100 & 100 \\
\hline Streptococcus viride & 100 & 100 & 100 & 100 & 100 & 100 & 100 \\
\hline
\end{tabular}

$500 \mathrm{mg} / \mathrm{d}$ ) had a CFR of $100 \%$ for methicillin-resistant Staphylococcus aureus, which was consistent with our results.

Although the concentration of dalbavancin decreased and itsclearance rate increased with the different grades of liver injury (grade B-C), the cytochrome P450 enzyme was not involved in the metabolism of dalbavancin and the drug concentration did not increase as the liver injury grade went up. So for patients with hepatic impairment, the results of PTA $>90 \%$ showed a MIC value was up to $2 \mu \mathrm{g} / \mathrm{mL}$. The study reported that dalbavancin was maintained at a dose greater than $20 \mathrm{mg} / \mathrm{L}$ during the 14-day clinical treatment of patients with hepatic injury, which was consistent with its antibacterial concentration in vivo. It suggested that the dose of dalbavancin may not be adjusted for patients with any levels of hepatic impairment.

For patients with severe renal insufficiency, the concentration of dalbavancin was significantly increased. Both the doses of $500 \mathrm{mg} / \mathrm{d}$ and $1000 \mathrm{mg} / \mathrm{d}$ can reach the target value. For the safety of the drug, the former dose can be selected for clinical treatment. In patients with end-stage renal disease (ESRD), although the clearance rate before and after drug dialysis was changed from small to large, the results of PTA $>90 \%$ showed that the MIC value was up to $0.5 \mu \mathrm{g} / \mathrm{mL}$. Moreover, it was reported that the concentration of dalbavancin was not detected in the dialysate of patients because of its large molecular weight resulting in the difficulty to move across the dialysis membrane. This indicates that dialysis in patients with ESRD has no effect on the metabolism of dalbavancin, thus no dose adjustment is needed during dialysis.

Although Monte Carlo simulation is hypothesized and analyzed based on certain PK and strainsand it is easy to optimize the types of antibacterial drugs and dose selection for treatment, the results of this study still have certain limitations. First of all, there is no complete unification of the target value of dalba- 
vancin fAUC24 h/MIC. For examples, it has been reported that the target value was set to either 292 [18] or 300 [14]. Secondly, we only considered the serum concentration drug when the Monte Carlo simulation was performed in this study. During the clinical trial, however, MIC values were evaluated from non-blood samples from sputum or effusion, which was not included in the present analyses of Monte Carlo simulation, further resulting in the inconsistent MIC values of the infected sample from different tissues. Finally, the source of bacterial MIC values is from the data of limited regions in previous years, so the results of this part of the samples are not suitable and do not reflect the changes in future trends.

\section{Conclusion}

In summary, our data demonstrate that the dose of dalbavancin in patients with hepatic and renal insufficiency simulated by MCS does not need to be adjusted. In clinical, patients with hepaticand renal dysfunction can obtain a certain reference according to MCS when using dalbavancin, but also using drugs in combination with clinical actual conditions.

\section{Funding}

This study was funded by the Study and Development Fund for Sciences and Technology in Chengde City (No. 201701A086).

\section{Data Availability Statement}

All data generated or analyzed during this study are included in this published article.

\section{Conflicts of Interest}

The authors declare no conflicts of interest regarding the publication of this paper.

\section{References}

[1] Garnock-Jones, K.P. (2017) Single-Dose Dalbavancin: A Review in Acute Bacterial Skin and Skin Structure Infections. Drugs, 77, 75-83. https://doi.org/10.1007/s40265-016-0666-0

[2] Meyer, B., Traunmueller, F., Hamwi, A., Hollenstein, U.M., Locker, G.J., Sperr, W.R., Staudinger, T., Thalhammer-Scherrer, R., Burgmann, H. and Thalhammer, F. (2004) Pharmacokinetics of Teicoplanin during Continuous Hemofiltration with a New and a 24-h Used Highly Permeable Membrane: Rationale for Therapeutic Drug Monitoring-Guided Dosage. International Journal of Clinical Pharmacology and Therapeutics, 42, 556-560. https://doi.org/10.5414/CPP42556

[3] Brown, N., Ho, D.H., Fong, K.L., Bogerd, L., Maksymiuk, A., Bolivar, R., Fainstein, V. and Bodey, G.P. (1983) Effects of Hepatic Function on Vancomycin Clinical Pharmacology. Antimicrobial Agents \& Chemotherapy, 23, 603-609. https://doi.org/10.1128/AAC.23.4.603

[4] Soon, R.L., Turner, S.J., Forrest, A., Tsuji, B.T. and Brown, J. (2013) Pharmacoki- 
netic/Pharmacodynamic Evaluation of the Efficacy and Safety of Daptomycin against Staphylococcus aureus. International Journal of Antimicrobial Agents, 42, 53-58. http://www.sciencedirect.com/science/article/pii/S0924857913000691 https://doi.org/10.1016/j.ijantimicag.2013.02.009

[5] Xu, G., Zhu, L., Ge, T., Liao, S., Li, N. and Qi, F. (2016) Pharmacokinetic/Pharmacodynamic Analysis of Voriconazole against Candida spp. and Aspergillus spp. in Children, Adolescents and Adults by Monte Carlo Simulation. International Journal of Antimicrobial Agents, 47, 439-445.

http://www.sciencedirect.com/science/article/pii/S0924857916300565

https://doi.org/10.1016/j.ijantimicag.2016.02.016

[6] Xie, J., Wang, T., Sun, J., Chen, S., Cai, J., Zhang, W., Dong, H., Hu, S., Zhang, D., Wang, X. and Dong, Y. (2014) Optimal Tigecycline Dosage Regimen Is Urgently Needed: Results from a Pharmacokinetic/Pharmacodynamic Analysis of Tigecycline by Monte Carlo Simulation. International Journal of Infectious Diseases, 18, 62-67. https://doi.org/10.1016/j.ijid.2013.09.008

[7] Mouton, J.W., Schmitt-Hoffmann, A., Shapiro, S., Nashed, N. and Punt, N.C. (2004) Use of Monte Carlo Simulations to Select Therapeutic Doses and Provisional Breakpoints of BAL9141. Antimicrobial Agents and Chemotherapy, 48, 1713-1718. https://www.ncbi.nlm.nih.gov/pubmed/15105125 https://www.ncbi.nlm.nih.gov/pmc/articles/PMC400534/ https://doi.org/10.1128/AAC.48.5.1713-1718.2004

[8] Doan, T.N., Kong, D.C.M., Patel, K., Walker, P., Spencer, A. and Kirkpatrick, C.M.J. (2014) Comparison of the Probability of Target Attainment of Anidulafungin against Candida spp. in Patients with Acute Leukaemia. International Journal of Antimicrobial Agents, 44, 450-457.

http://www.sciencedirect.com/science/article/pii/s0924857914002556

https://doi.org/10.1016/j.ijantimicag.2014.07.019

[9] Xu, G., Zhu, L., Liao, S., Ge, T. and Yang, J. (2015) Assessment of Echinocandin Regimens by Pharmacokinetic/Pharmacodynamic Analysis against Candida spp. in Paediatric Patients. International Journal of Antimicrobial Agents, 46, 631-641. http://www.sciencedirect.com/science/article/pii/S0924857915003131 https://doi.org/10.1016/j.ijantimicag.2015.08.009

[10] Thomas, M., Dowell, J.A., Elyse, S. and Mary, B. (2013) Pharmacokinetics of Dalbavancin in Patients with Renal or Hepatic Impairment. Journal of Clinical Pharmacology, 49, 465-476. https://doi.org/10.1177/0091270008330162

[11] Jones, R.N., Sader, H.S. and Flamm, R.K. (2013) Update of Dalbavancin Spectrum and Potency in the USA: Report from the SENTRY Antimicrobial Surveillance Program (2011). Diagnostic Microbiology and Infectious Disease, 75, 304-307. https://doi.org/10.1016/j.diagmicrobio.2012.11.024

[12] David, A. and Craig, W.A. (2007) In Vivo Pharmacodynamic Activity of the Glycopeptide Dalbavancin. Antimicrobial Agents and Chemotherapy, 51, 1633-1642. https://doi.org/10.1128/AAC.01264-06

[13] Nasia, S., David, A. and Craig, W.A. (2004) In Vivo Pharmacodynamic Activity of Daptomycin. Antimicrobial Agents and Chemotherapy, 48, 63-68. https://doi.org/10.1128/AAC.48.1.63-68.2004

[14] Dowell, J.A., Goldstein, B.P., Mary, B., Martin, S. and Bharat, D. (2013) Pharmacokinetic-Pharmacodynamic Modeling of Dalbavancin, a Novel Glycopeptide Antibiotic. Journal of Clinical Pharmacology, 48, 1063-1068.

https://doi.org/10.1177/0091270008321273 
[15] Smith, J.R., Roberts, K.D. and Rybak, M.J. (2015) Dalbavancin: A Novel Lipoglycopeptide Antibiotic with Extended Activity Against Gram-Positive Infections. Infectious Diseases \& Therapy, 4, 245-258. https://doi.org/10.1007/s40121-015-0077-7

[16] Anton, L., Alice Bendix, G., Mary Beth, D., Daniela, J., Giorgio, M., Claudia, V.S., Mroszczak, E.J., Campbell, K.C.M. and Ellen, K. (2004) Tolerability, Pharmacokinetics, and Serum Bactericidal Activity of Intravenous Dalbavancin in Healthy Volunteers. Antimicrobial Agents \& Chemotherapy, 48, 940-945.

https://doi.org/10.1128/AAC.48.3.940-945.2004

[17] Bai, F.D.X. (2015) Dabavancin, a New Glycopeptide Antibiotic. Chinese Journal of New Drugs, 24, 1441-1444.

[18] Ahmed Hamed, S., Zhanel, G.G., Ibrahim, S.A. and Noreddin, A.M. (2014) Monte Carlo Simulation Analysis of Ceftobiprole, Dalbavancin, Daptomycin, Tigecycline, Linezolid and Vancomycin Pharmacodynamics against Intensive Care Unit-Isolated Methicillin-Resistant Staphylococcus aureus. Clinical \& Experimental Pharmacology \& Physiology, 41, 437-443. https://doi.org/10.1111/1440-1681.12195 